\title{
Layer-specific strain and dyssynchrony index alteration in new-onset systemic lupus erythematosus patients without cardiac symptoms
}

\author{
Tingting Luo ${ }^{1 \#}$, Zhenhua Wang $^{2 \#}$, Zhen Chen $^{3}$, Ermei Yu ${ }^{1}$, Chenglong Fang ${ }^{3}$ \\ ${ }^{1}$ Department of Echocardiography, Second Affiliated Hospital of Fujian Medical University, Quanzhou, China; ${ }^{2}$ Department of Cardiology, Second \\ Affiliated Hospital of Fujian Medical University, Quanzhou, China; ${ }^{3}$ Department of Rheumatology, Second Affiliated Hospital of Fujian Medical \\ University, Quanzhou, China
}

\#These authors contributed equally to this work.

Correspondence to: Chenglong Fang. Department of Rheumatology, Second Affiliated Hospital of Fujian Medical University, Donghai Avenue 950, Quanzhou 362000, China. Email: fangc1900523@126.com.

Background: Layer-specific speckle-tracking echocardiography (STE) is a noninvasive approach that assesses subclinical left ventricular dysfunction. We aimed to investigate the (I) alteration of layer-specific STE parameters and the dyssynchrony index; and (II) the disease parameters associated with layer-specific STE change in drug-naïve patients with new-onset systemic lupus erythematosus (SLE) without cardiac symptoms.

Methods: Thirty-five drug-naïve patients with new-onset SLE and twenty-five healthy controls were enrolled. All individuals received both conventional echocardiographic and two-dimensional STE assessment. The data of layer-specific global longitudinal strain (GLS), global circumferential strain (GCS), and peak systolic dispersion (PSD) were acquired in layer-specific STE.

Results: All patients had a normal left ventricular ejection fraction (LVEF)(mean LVEF: 58\%) and conventional echocardiographic parameters were comparable between patients and controls. Decreased layer-specific GLS and elevated PSD were observed in SLE patients (whole layer GLS: $-17.6 \% \pm 3.0 \%$ versus $-19.3 \% \pm 2.6 \%, \mathrm{P}=0.02$; endocardial GLS: $-20.0 \% \pm 3.2 \%$ versus $-22.1 \% \pm 3.0 \%, \mathrm{P}=0.01$; epicardial GLS: $-15.6 \% \pm 2.7 \%$ versus $-16.8 \% \pm 2.4 \%, \mathrm{P}=0.04$; PSD: $41.0 \pm 18.9$ versus $28.8 \pm 10.1 \mathrm{msec}, \mathrm{P}=0.007)$. In contrast, there was no difference in layer-specific GCS at three different levels between patients and controls $(\mathrm{P}>0.05)$. More severely impaired GLS was observed in patients with higher disease activity, high-risk antiphospholipid antibody (aPL) profile, or renal involvement. The PSD was increased in patients with higher disease activity or a high-risk aPL profile. Correlational analysis showed that GLS at three layers and PSD correlated with high-sensitivity C-reactive protein (hsCRP) levels (whole GLS: $\mathrm{r}=0.662, \mathrm{P}<0.001$; endocardial GLS: $\mathrm{r}=0.637$, $\mathrm{P}<0.001$; epicardial GLS: $\mathrm{r}=0.658, \mathrm{P}<0.001$; PSD: $\mathrm{r}=0.390, \mathrm{P}=0.021)$. PSD correlated with epicardial GLS $(\mathrm{r}=0.360, \mathrm{P}=0.047)$, when treating the hsCRP level, renal involvement, aPL profile, and disease activity as control variables. Multivariate regression showed the hsCRP level and epicardial GLS were predictors of layer-specific GLS impairment and elevated PSD, respectively.

Conclusions: Drug-naive patients with new-onset SLE are likely to have subclinical GLS impairment and left ventricular dyssynchrony, even in the presence of normal LVEF. SLE-related risk factors are associated with these dysfunctions.

Keywords: Layer-specific speckle-tracking echocardiography (layer-specific STE); left ventricular dyssynchrony; systemic lupus erythematosus (SLE) 
Submitted Jul 13, 2020. Accepted for publication Oct 26, 2020.

doi: 10.21037/qims-20-859

View this article at: http://dx.doi.org/10.21037/qims-20-859

\section{Introduction}

With advances in immunosuppressive therapies, the shortterm outcome of patients with systemic lupus erythematosus (SLE) has significantly improved. However, SLE patients have up to a 10 -fold increased risk of cardiovascular morbidity and mortality, and cardiac involvement still accounts for most deaths (1). Cardiac impairment in SLE is often asymptomatic and not always in parallel with disease activity $(2,3)$. While transthoracic echocardiography is a routine method to screen cardiovascular system involvement in SLE, it often fails to detect subclinical ventricular dysfunction for several reasons. For example, abnormal left ventricular ejection fraction (LVEF) tends to reflect global but not regional myocardial dysfunction. Furthermore, disadvantages including load and angle dependency and low reproducibility exist in the measurement of LVEF (4).

Speckle-tracking echocardiography (STE) is a practical and noninvasive method of assessing cardiac function which has attracted increased attention in the past two decades. While STE provides important information on subclinical left ventricular dysfunction $(5,6)$, layer-specific STE has the advantage of quantifying longitudinal-and circumferentiallayer strains of the endocardium, midmyocardium, and epicardium, which makes it more sensitive and accurate than STE (7). Moreover, peak systolic dispersion (PSD) acquired in layer-specific STE and defined as the standard deviation of the time to peak strain of the 18 segments, reflects left ventricular mechanical dyssynchrony (LVMD). Patients with LVMD have a poor prognosis, especially those with preserved ejection fraction $(8,9)$.

To the best of our knowledge, the present study is the first to evaluate silent cardiac dysfunction and mechanical dyssynchrony in drug-naive patients with new-onset SLE. Disease parameters associated with layer-specific STE change were also explored.

\section{Methods}

\section{Study subjects}

Drug-naive patients with new-onset SLE were consecutively recruited from the Second Affiliated Hospital of Fujian Medical University from January 2019 to January
2020. All patients fulfilled the 2012 American College of Rheumatology (ACR)/Systemic Lupus International Collaborating Clinics (SLICC) criteria (10). The onset of SLE-related symptoms occurred within 1 month prior to study entry, and patients had no cardiac symptoms. Disease activity was assessed by the Systemic Lupus Erythematosus Disease Activity Index-2K (SLEDAI-2K). The high-risk antiphospholipid antibody (aPL) profile was defined as the presence of lupus anticoagulant, or of double or triple aPL positivity, or the presence of persistently high aPL titres (11). A group of healthy controls included agematched volunteers with a negative result to antinuclear antibodies and no history of systemic disease was also established. Other inclusion criteria for this group were normal echocardiography, electrocardiographic, N-terminal pro-B-type natriuretic peptide (NT-proBNP), and troponin I results. Exclusion criteria included age $<18$ years, a history of treatment with cardio-toxic drugs, acute coronary symptoms or symptomatic heart failure, previous cardiac surgery, obesity, hypertension, smoking, diabetes, bundlebranch heart block, an estimated glomerular filtration rate of $<30 \mathrm{~mL} /$ minute $/ 1.73 \mathrm{~m}^{2}$, and tumor. This study was approved by the ethics committee of the Second Affiliated Hospital of Fujian Medical University. Written informed consent forms were obtained from all participants.

\section{Routine echocardiographic assessment}

We performed two-dimensional (2D) echocardiography examinations using a GE VIVID E95 ultrasound system equipped with a $1.4-4.6 \mathrm{MHz}$ transducer (M5Sc-D probe). The LVEF was measured by the modified Simpson's method and determined using the following parameters: left ventricular end-diastolic diameters (LVEDD), left ventricular end-systolic diameters (LVESD), interventricular septal (IVS), posterior wall thickness (PWT), left ventricular end-diastolic volume (LVEDV), and left ventricular end-systolic volumes (LVESV). Diastolic parameters included E, A, septal e', lateral e', E/e', tricuspid regurgitation (TR) velocity, and left atrial volume index (LAVI). The "E" represents the trans-mitral peak velocity of early diastole, while the "e" and the "A" refer to the velocity of early diastole of the mitral annulus 
and trans-mitral peak velocity of late diastole, respectively. Left ventricular diastolic function was assessed according to the 2016 American Society of Echocardiography/ European Association of Cardiovascular Imaging recommendations (12). In patients with normal LVEF, the four variables for identifying diastolic dysfunction, and their abnormal cutoff values were the following: septal e' $<7 \mathrm{~cm} / \mathrm{sec}$, lateral e' $<10 \mathrm{~cm} / \mathrm{sec}$, average $\mathrm{E} / \mathrm{e}^{\prime}$ ratio $>14$, LAVI $>34 \mathrm{~mL} / \mathrm{m} 2$, and peak TR velocity $>2.8 \mathrm{~m} / \mathrm{sec}$.

\section{D STE assessment}

Two-dimensional STE was performed using a GE VIVID E95 ultrasound system. Images of the apical four-chamber, apical two-chamber, apical LV long-axis, and LV shortaxis views at the basal, papillary, and apical levels were collected within 3-5 cardiac cycles. The software package EchoPAC (version 201 6.3, GE Vingmed Ultrasound) was used to analyze images. Software tracked the endocardium outline and divided the whole ventricle wall into three layers (an endocardial, a mid-myocardial and an epicardial layer). Quantitative myocardial parameters for the global longitudinal strain (GLS) and global circumference strain (GCS) of each layer, and PSD, were obtained. STE data analyses were conducted by two investigators (TL and ZW) who had experience with layer-specific STE and were blind to the clinical data.

\section{Statistical analysis}

Differences and correlations between patients and controls were analyzed using $t$-test and Pearson's correlation analysis, respectively, when the data was normally distributed. When it was not, the nonparametric Mann-Whitney rank-sum test and Spearman's correlation analysis were applied. Partial correlation analysis and multivariate linear regression were used to find the disease parameters associated with abnormal measure values in STE. Inter- and intra-observer reproducibility was assessed using intraclass correlation coefficients. Statistical significance was defined as $\mathrm{P}<0.05$, and all statistical analyses were performed with GraphPad Prism software 5.0 and SPSS software 21.0.

\section{Results}

\section{Study population}

The characteristics of SLE patients and healthy controls are summarized in Table 1. A total of 35 SLE patients with newonset disease and 25 healthy controls were enrolled. Stableto-mild disease activity was found in $51 \%$ of patients $(\mathrm{n}=18)$, while $40 \%$ had moderate disease activity ( $\mathrm{n}=14)$, and $9 \%$ had high disease activity ( $\mathrm{n}=3)$. No cardiac symptoms were reported in either SLE patients or controls. All participants had normal serum markers of cardiac injury including $\mathrm{N}$-terminal pro-B-type natriuretic peptide (NT-proBNP) and troponin I, indicating an absence of clinical evidence of cardiac impairment in the SLE patients.

\section{Conventional echocardiographic parameters}

Conventional echocardiographic results are summarized in Table 2. All participants had normal LVEF. No significant differences of parameters assessing systolic and diastolic function including LVEDV, LVESV, LVEDD, LVESD, IVS, PWT, E, A, septal e', lateral e', averaged e', E/e', TR velocity, or LAVI were found between SLE patients and healthy controls $(\mathrm{P}>0.05$, all $)$.

\section{Layer-specific STE characteristics, left ventricular dyssynchrony index (PSD), and disease parameters in SLE patients}

The representative images of layer-specific STE's of one SLE patient and one healthy control are shown in Figures 1 and 2 respectively, and the results of layerspecific STE parameters are displayed in Table 3. Although clinical evidence of cardiac involvement was not detected in SLE patients through routine assessment, an increase in impairment of left ventricular GLS at three layers (whole layer GLS, endocardial GLS, and epicardial GLS) was observed in drug-naïve patients with new-onset SLE, compared with controls ( $\mathrm{P}=0.02,0.01,0.04$, respectively). Elevation of PSD, which reflects left ventricular dyssynchrony, was also found in SLE patients $(\mathrm{P}<0.01)$. In contrast, there was no significant difference in the GCS of the three layers (midmyocardium GCS, endocardium GCS and epicardium GCS) at the basal, papillary, and apical levels between SLE patients and controls $(\mathrm{P}>0.05$, all).

In addition to the above findings, more severe impairment of GLS was observed in SLE patients with moderate-to-severe active disease, high-risk aPL profile, or lupus nephritis, compared with those with inactive-tomild active disease, negative-to-low-risk aPL profile, or no evidence of renal involvement, respectively (Table 4). In contrast, PSD was significantly increased in patients with 
Table 1 Characteristics of SLE patients and controls

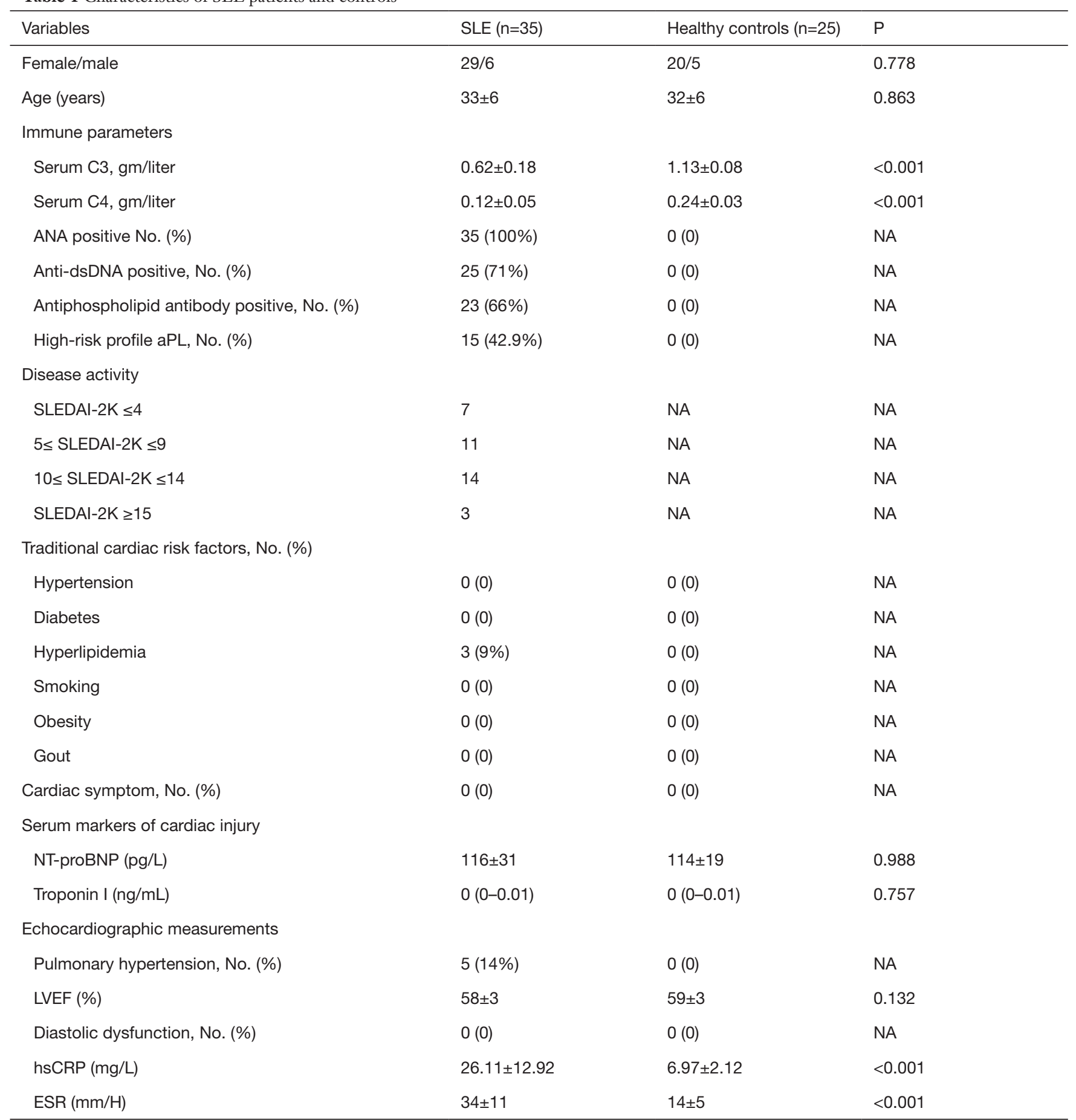

ANA, antinuclear antibody; anti-dsDNA, anti-double-stranded DNA; SLEDAI-2K, Systemic Lupus Erythematosus Disease Activity Index2K; aPL, antiphospholipid antibody; NT-proBNP, n-terminal pro-b-type natriuretic peptide; LVEF, left ventricular ejection fraction; hsCRP, high sensitivity C-reactive protein; ESR, erythrocyte sedimentation rate 
Table 2 Conventional and TDI echocardiographic parameters in SLE patients and controls

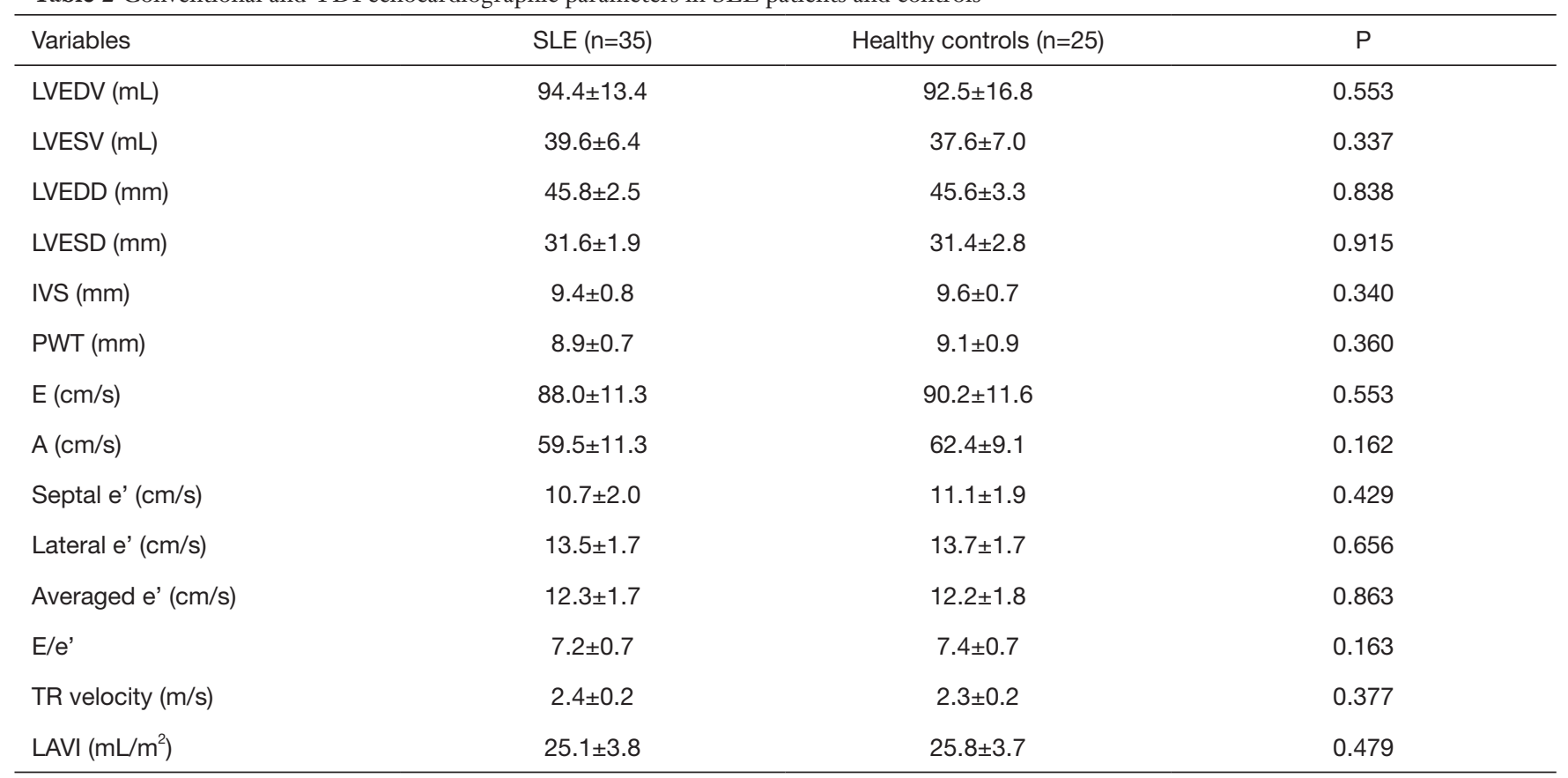

LVEDV, left ventricular end-diastolic volume; LVESV, left ventricular end-systolic volume; LVEDD, left ventricular end-diastolic diameter; LVESD, left ventricular end-systolic diameter; IVS, interventricular septum; PWT, posterior wall thickness; E, transmitral peak velocity of early diastole; A, transmitral peak velocity of late diastole; e', velocity of early diastole of the mitral annulus; TR velocity, tricuspid regurgitation velocity; LAVI, left atrial volume index.

higher disease activity or high-risk aPL, but not in those with lupus nephritis (Table 4).

Correlation analysis showed that endocardial GLS, whole layer GLS, epicardial GLS, and PSD correlated with highsensitivity CRP (hsCRP) levels in SLE patients, but not in healthy controls. PSD also correlated with the three layerspecific GLS parameters above (Figure 3). Partial correlation analysis showed that PSD correlated with epicardial GLS, but not with whole layer GLS or endocardial GLS, when treating hsCRP level, renal involvement, aPL profile, and disease activity as control variables (Table 5). Multivariate regression analysis showed that hsCRP level and epicardial GLS are the predictors of layer-specific GLS impairment and PSD change, respectively (Table 6).

\section{Intra- and inter-observer variability of GLS}

Intra-observer variabilities and inter-observer reliability for whole GLS, endocardial GLS, and epicardial GLS were analyzed by a Bland-Altman plot presented (Figure 4), and were all within the limits of agreement.

\section{Discussion}

In 2016, Ozawa et al. first analyzed the relationship between layer-specific strain parameters and heart failure indicators in 20 systemic autoimmune disorder patients, including 7 SLE patients. They found that GCS values, but not GLS in whole, endocardial, or epicardial layers, correlated with serum B-type natriuretic peptide (BNP) levels (13). In addition, GCS in the endocardial layer was significantly positively correlated with LVEF. To the best of our knowledge, the present study is the first to examine asymptomatic myocardial dysfunction in drug-naïve patients with new-onset SLE via a speckle-tracking-based multilayer approach. The main findings are that decreased whole layer GLS, endocardial GLS, and epicardial GLS could be found in SLE patients, when compared with age- and sexmatched healthy individuals. This suggests that subclinical impairment of left ventricular function can occur as early as the time when SLE is newly diagnosed and LVEF remains preserved.

Interestingly, SLE patients had impaired myocardial GLS but not GCS. The relationship between LS and CS is 

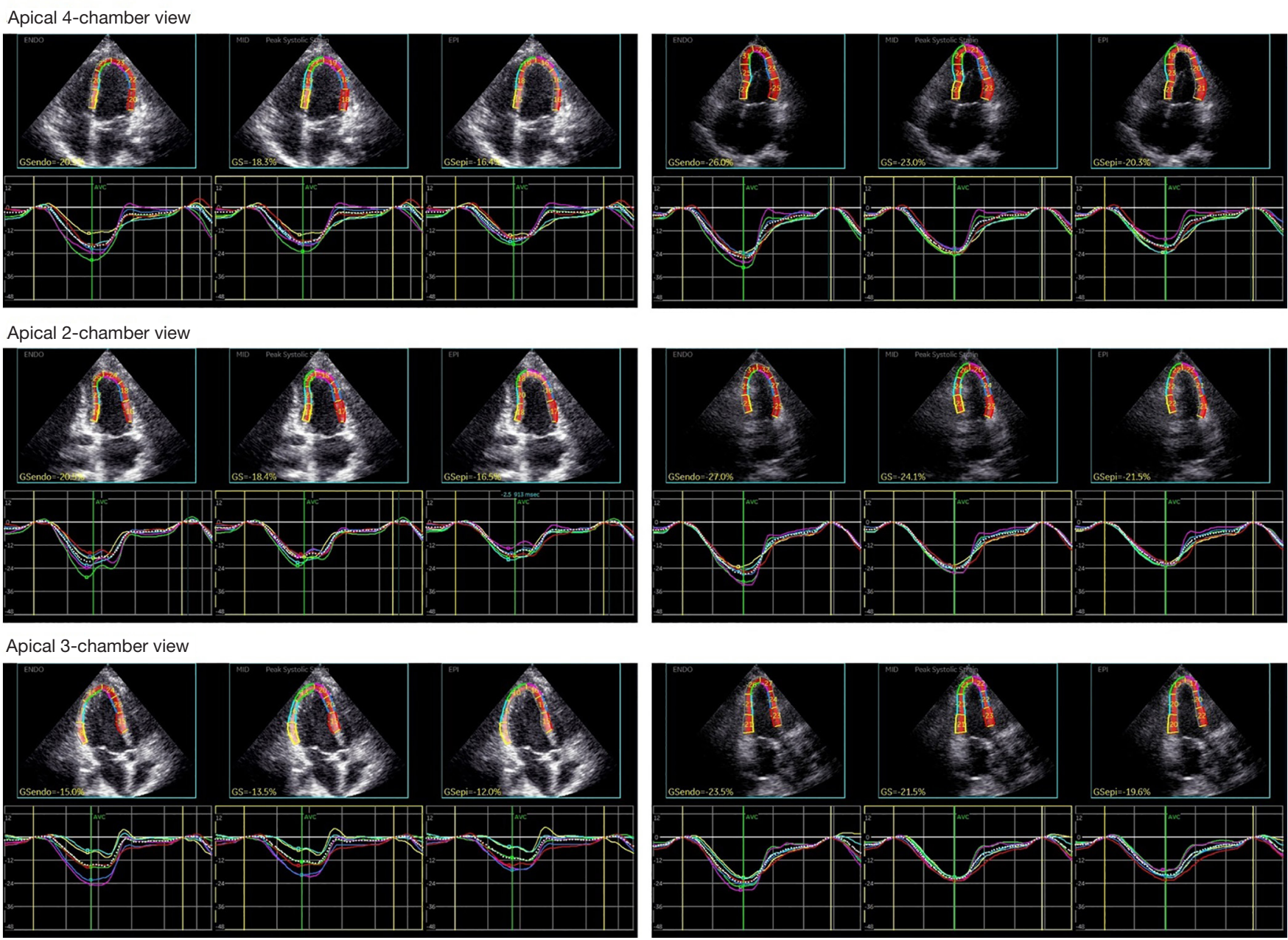

Figure 1 Typical layer-specific global longitudinal strain (GLS) images of the left ventricle (LV) from a patient with systemic lupus erythematosus (left) and a healthy individual (right). LV GLS was measured from three different views [apical 4 (upper level images)-, 2 (middle level images)-, and 3 (bottom level images)-chamber views]. Measurement of whole layer GLS, endocardial GLS, and epicardial GLS were performed.

still not clear. Emerging evidence shows that different roles are played by CS and LS in left ventricular ejection (14), with circumferential shortening contributing more to LVEF $(14,15)$. In contrast, patients with impaired longitudinal shortening constantly have preserved LVEF. This result was also consistent with the study by Kraigher-Krainer et al. who found that in heart failure with preserved ejection fraction (HFpEF), after adjustment for 10 baseline covariates, lower LS but not CS was associated with higher NT-proBNP, a prognostically relevant biomarker in HFpEF (16). In our study, decreased GLS was found in new-onset SLE patients with normal LVEF, suggesting GLS might be a marker for the early detection of myocardial dysfunction in SLE patients.

PSD has been proven to be a relatively new and useful marker for the early detection of $\operatorname{LVMD}(17,18)$. Mounting evidence suggests that LVMD has an independent and incremental negative impact on myocardial mechanics and remodeling. In the study by Leong et al., left ventricular (LV) dyssynchrony was independently associated with changes in the LVEF over time in idiopathic dilated cardiomyopathy and the occurrence of ventricular tachycardia after myocardial infarction (19). Fudim et al. demonstrated that LV dyssynchrony was strongly associated with adverse outcomes among patients with coronary heart disease (20). In our study, elevation of PSD was detected 
GCS at apical level
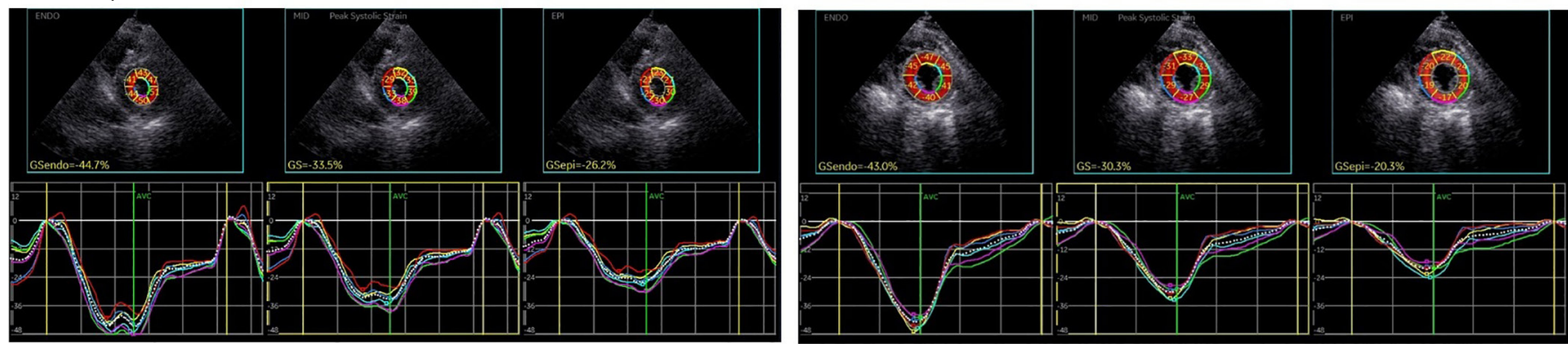

GCS at papillary muscle level
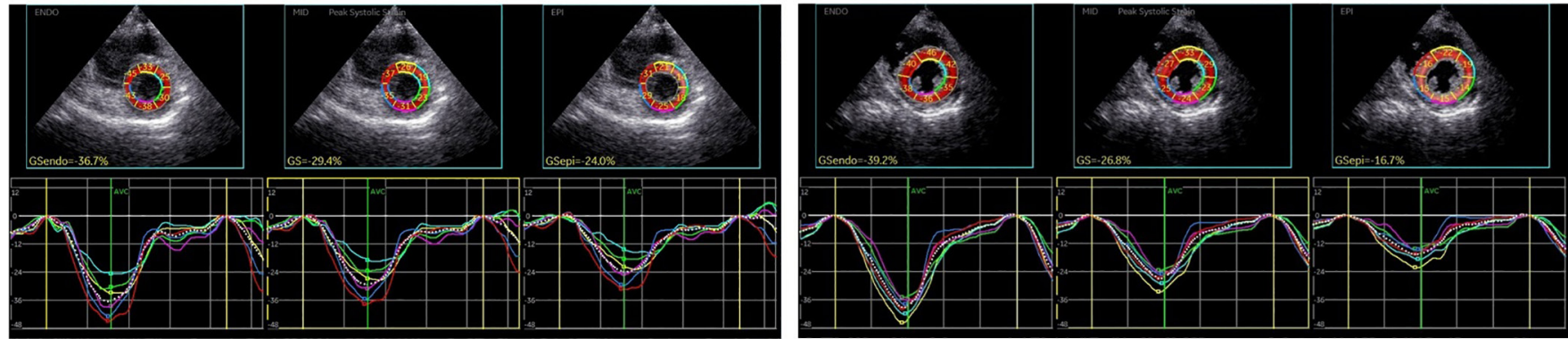

GCS at basal level
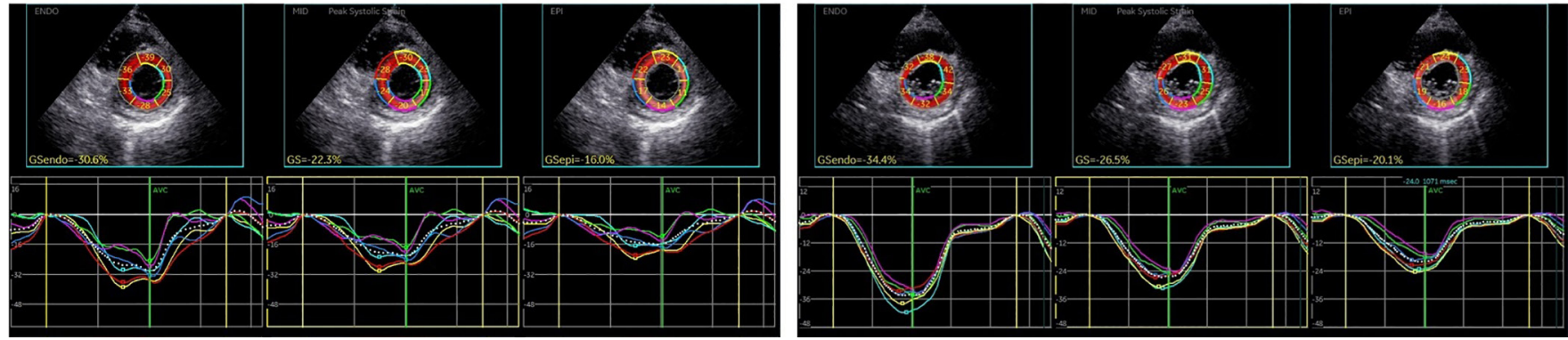

Figure 2 Representative plot of layer-specific global circumferential strain (GCS) in the left ventricle (LV) of an SLE patient (left) and a healthy individual (right). LV GCS was measured from three different levels (apical level, papillary muscle level, and basal level). Measurement of midmyocardium GCS, endocardium GCS, and epicardium GCS were performed.

in drug-naïve SLE patients for the first time, suggesting subclinical LVMD in SLE. Moreover, previous studies have shown that LVMD was particularly prevalent in patients with microvascular involvement (21), which is also a characteristic lesion mediated by immune complex deposition in SLE. Whether or not higher PSD predicts a poorer myocardial prognosis in patients with SLE needs to be clarified in further research.

Both mouse models and clinical evidence have shown that lupus-associated risk factors play key roles in the pathogenesis of cardiovascular disease (CVD) in SLE patients $(22,23)$. Disease activity, inflammation, antiphospholipid antibody, and lupus nephritis are among the most studied SLE-associated cardiovascular risk factors
(24-26). Similarly, our results showed that more active disease, high-risk aPL profile, renal involvement, and higher hsCRP level were linked with the tendency towards more severe; 7 impaired GLS parameters and higher PSD (although not always with statistical significance). Active disease is associated with macrophage activation, which enhances the pro-inflammatory process of CVD occurrence in SLE (27). A high-risk aPL profile not only mediates hypercoagulable states and endothelial dysfunction, but also exacerbates atherosclerosis inflammation in SLE with antiphospholipid syndrome (28). Gustafsson et al. reported that accelerated atherosclerosis in SLE is mainly confined to a subgroup with lupus nephritis (29). The four factors above may work alone or coordinate to cause LS and PSD 
Table 3 Layer-specific strain data and left ventricular dyssynchrony index parameters in SLE patients and controls

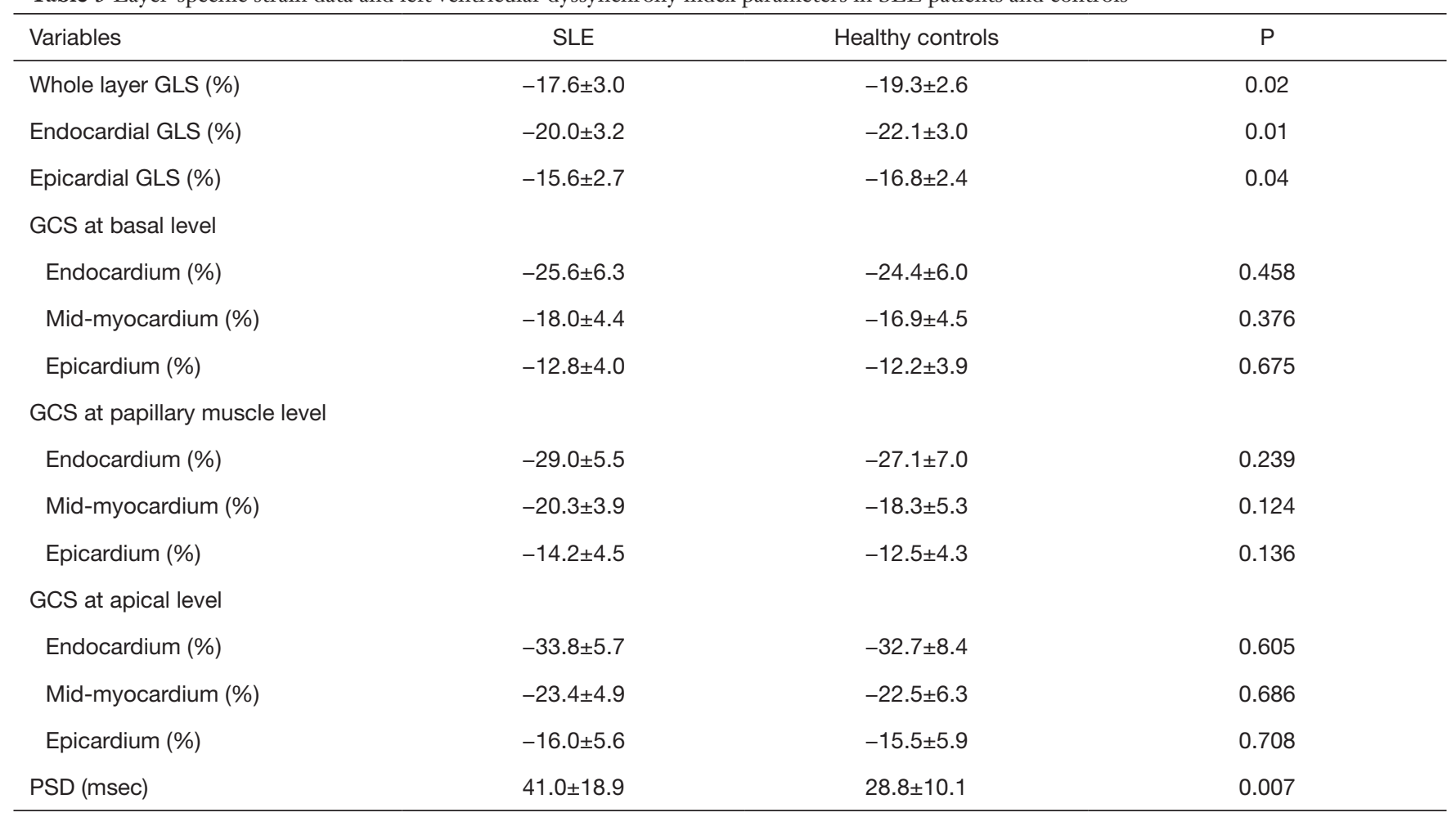

STE, speckle-tracking echocardiography; GCS, global circumferential strain; GLS, global longitudinal strain; PSD, peak systolic dispersion.

Table 4 Layer-specific longitudinal parameters, PSD, and certain disease-related contributors of cardiovascular risk in SLE patients

\begin{tabular}{|c|c|c|c|c|}
\hline Variable & Whole layer GLS (\%) & Endocardial GLS (\%) & Epicardial GLS (\%) & PSD (msec) \\
\hline \multicolumn{5}{|l|}{ Disease activity } \\
\hline Inactive-to-mild active disease & $-19.3 \pm 2.3$ & $-21.8 \pm 2.6$ & $-17.1 \pm 2.2$ & $34.1 \pm 16.1$ \\
\hline $\begin{array}{l}\text { Moderate-to-severe active } \\
\text { disease }\end{array}$ & $-15.8 \pm 2.5$ & $-18.1 \pm 2.8$ & $-13.9 \pm 2.3$ & $48.2 \pm 19.3$ \\
\hline Without lupus nephritis & $-19.5 \pm 2.1$ & $-22.0 \pm 2.4$ & $-17.4 \pm 2.0$ & $35.1 \pm 17.4$ \\
\hline With lupus nephritis & $-16.6 \pm 2.9$ & $-19.0 \pm 3.2$ & $-14.6 \pm 2.6$ & $44.0 \pm 19.3$ \\
\hline $\mathrm{P}$ & $<0.001$ & 0.001 & 0.002 & 0.062 \\
\hline \multicolumn{5}{|l|}{ aPL profile } \\
\hline High-risk aPL profile & $-15.9 \pm 2.8$ & $-18.3 \pm 3.2$ & $-14.0 \pm 2.5$ & $47.1 \pm 18.4$ \\
\hline$P$ & 0.002 & 0.002 & 0.004 & 0.037 \\
\hline
\end{tabular}

GCS, global circumferential strain; GLS, global longitudinal strain; PSD, peak systolic dispersion; aPL, antiphospholipid antibody. 

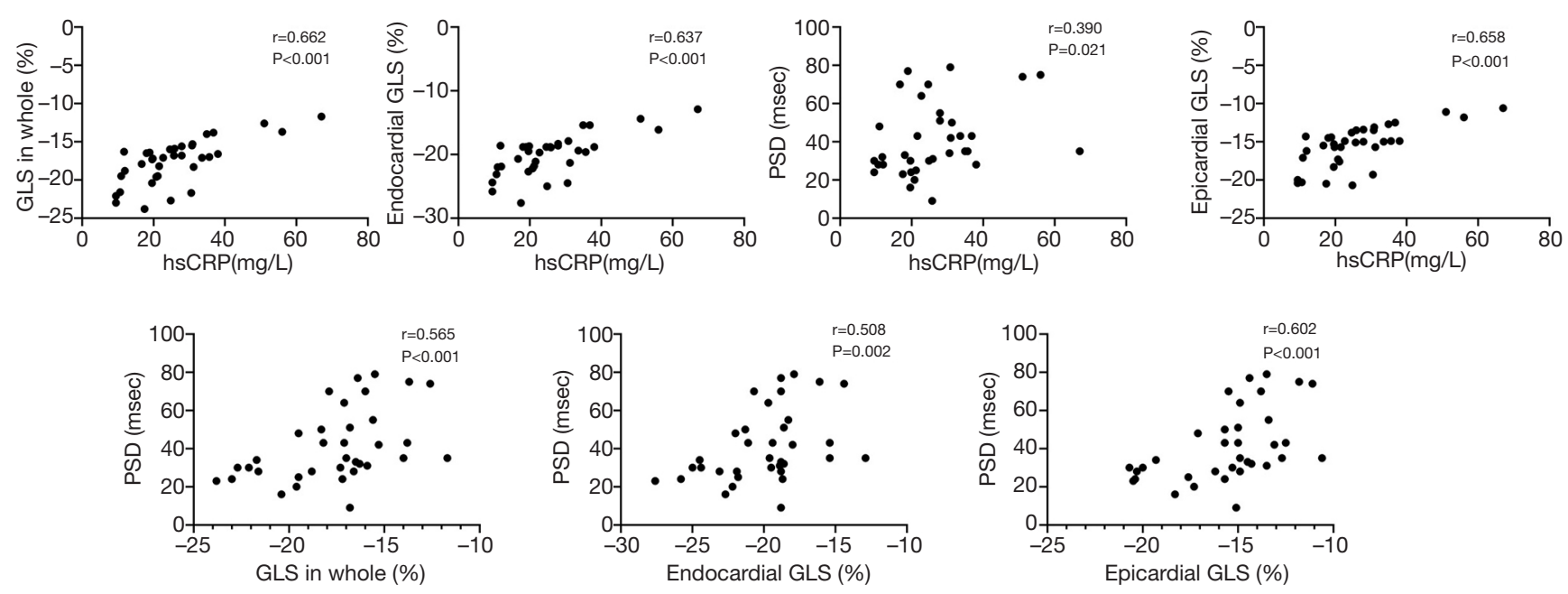

Figure 3 Univariate correlation between (I) global longitudinal strain (GLS) parameters and high sensitivity C reactive protein (hsCRP) levels; (II) peak systolic dispersion (PSD) and hsCRP levels; (III) PSD and GLS parameters in SLE patients.

Table 5 Partial correlation analysis between PSD and layer-specific GLS parameters

\begin{tabular}{lccc}
\hline Control variable & Variable (between PSD and below) & $r$ & P \\
\hline HsCRP level & Whole layer GLS & 0.296 & 0.106 \\
Renal involvement & Endocardial GLS & 0.212 & 0.252 \\
Profile of aPL & Epicardial GLS & 0.360 & 0.047 \\
Disease activity & & & \\
\hline
\end{tabular}

PSD, peak systolic dispersion; GLS, global longitudinal strain; hsCRP, high sensitivity C-reactive protein; aPL, antiphospholipid antibody.

impairment. Interestingly, PSD correlated with the threelayer-specific GLS parameters in the univariate analysis. Furthermore, partial correlation and multivariate regression analysis revealed that epicardial GLS is an independent predictor of PSD, suggesting that epicardial GLS may be a contributor to LVMD.

Routine echocardiography is commonly used to assess cardiac dysfunction in SLE patients. However, serious limitations exist for the reasons referred to above. In this study, all participants had normal left ventricular systolic and diastolic function on routine echocardiography, suggesting the longitudinal parameters measured in STE are more sensitive tools for assessing myocardial function impairment at an early stage.

There are some limitations to this study. Firstly, the predictive value of layer-specific STE parameters could not be judged well. Secondly, we did not enroll SLE patients having cardiac symptoms as the first manifestation to assess the clinical significance more comprehensively. A large-scale study with long-term follow-up in SLE patients is needed to evaluate the prognostic value of the STE parameters. Finally, we could not exclude the possibility that pulmonary hypertension $(\mathrm{PH})$ contributes to impaired GLS in SLE patients. The limited number of patients with SLE-PH in this study makes it difficult to perform a subgroup analysis between SLE-PH and SLE-non-PH patients. A further study will need to include a larger cohort of patients to clarify this issue.

\section{Conclusions}

This is the first study to demonstrate that drug-naive patients with new-onset SLE, including those with normal LVEF, are likely to have subclinical GLS impairment and left ventricular dyssynchrony. Some SLE-related risk factors are associated with these dysfunctions. 
Table 6 Multivariate linear regression to analyze predictors of layer-specific GLS parameters and PSD

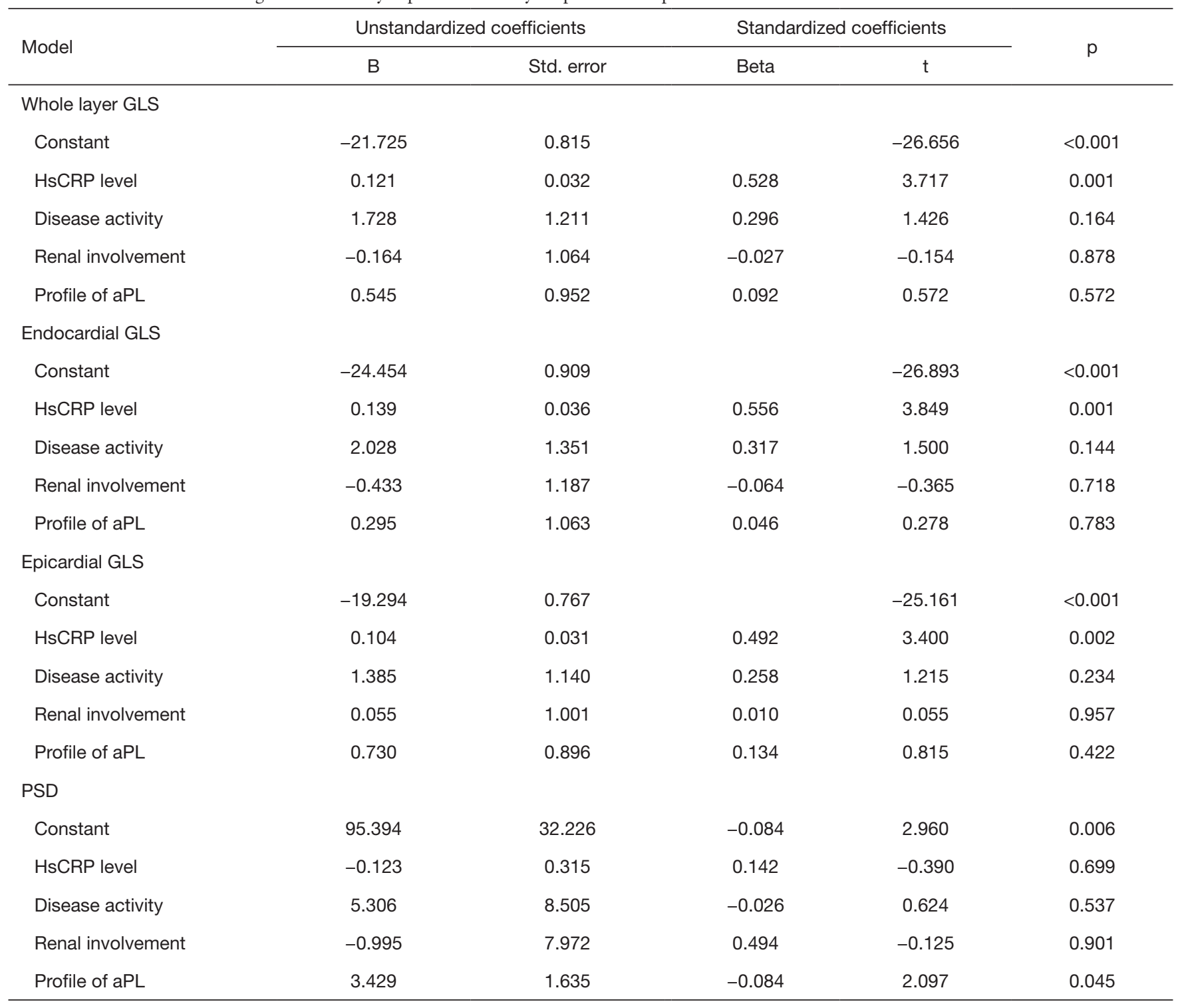

GLS, global longitudinal strain; PSD, peak systolic dispersion; hsCRP, high sensitivity C-reactive protein; aPL, antiphospholipid antibody. 

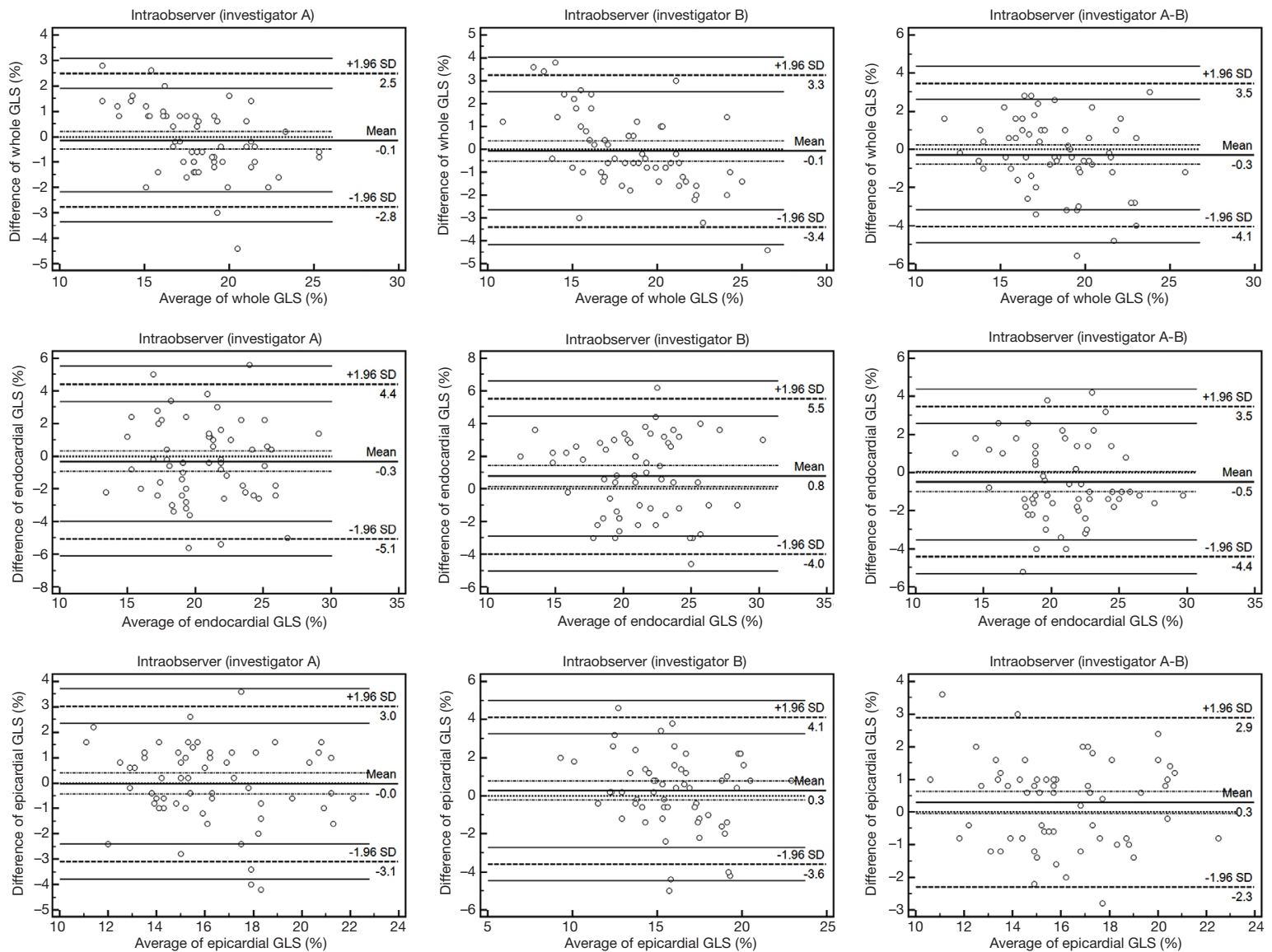

Figure 4 Bland-Altman analysis of (I) intra-observer reliability for whole global longitudinal strain (GLS), endocardial GLS, and epicardial GLS of two investigators (left and middle); (II) interobserver reliability of whole GLS, endocardial GLS, and epicardial GLS (right).

\section{Acknowledgments}

Funding: This work was supported by Key Clinical Specialty Discipline Construction Program of Fujian, China, Natural Science Foundation of Fujian Province (grant No. 2019J01473) and the Science and Technology Program of Quanzhou (grant No. 2018N014S, 2017Z009).

\section{Footnote}

Conflicts of Interest: All authors have completed the ICMJE uniform disclosure form (available at http://dx.doi. org/10.21037/qims-20-859). The authors have no conflicts of interest to declare.

Ethical Statement: Written informed consent forms were obtained from all individuals included. This study was approved by the ethics committee of the Second Affiliated
Hospital of Fujian Medical University.

Open Access Statement: This is an Open Access article distributed in accordance with the Creative Commons AttributionNonCommercial-NoDerivs 4.0 International License (CC BYNC-ND 4.0), which permits the non-commercial replication and distribution of the article with the strict proviso that no changes or edits are made and the original work is properly cited (including links to both the formal publication through the relevant DOI and the license). See: https://creativecommons. org/licenses/by-nc-nd/4.0/.

\section{References}

1. Thomas G, Mancini J, Jourde-Chiche N, Sarlon G, Amoura Z, Harlé JR, Jougla E, Chiche L. Mortality associated with systemic lupus erythematosus in France assessed by multiple-cause-of-death analysis. Arthritis Rheumatol 
2014;66:2503-11.

2. Bernatsky S, Boivin JF, Joseph L, Manzi S, Ginzler E, Gladman DD, Urowitz M, Fortin PR, Petri M, Barr S, Gordon C, Bae SC, Isenberg D, Zoma A, Aranow C, Dooley MA, Nived O, Sturfelt G, Steinsson K, Alarcón G, Senécal JL, Zummer M, Hanly J, Ensworth S, Pope J, Edworthy S, Rahman A, Sibley J, El-Gabalawy H, McCarthy T, St Pierre Y, Clarke A, Ramsey-Goldman R. Mortality in systemic lupus erythematosus. Arthritis Rheum 2006;54:2550-7.

3. Guo Q, Wu LM, Wang Z, Shen JY, Su X, Wang CQ, Gong XR, Yan QR, He Q, Zhang W, Xu JR, Jiang M, Pu J. Early Detection of Silent Myocardial Impairment in Drug-Naive Patients With New-Onset Systemic Lupus Erythematosus: A Three-Center Prospective Study. Arthritis Rheumatol 2018;70:2014-24.

4. Mor-Avi V, Lang RM, Badano LP, Belohlavek M, Cardim NM, Derumeaux G, Galderisi M, Marwick T, Nagueh SF, Sengupta PP, Sicari R, Smiseth OA, Smulevitz B, Takeuchi M, Thomas JD, Vannan M, Voigt JU, Zamorano JL. Current and evolving echocardiographic techniques for the quantitative evaluation of cardiac mechanics: ASE/ EAE consensus statement on methodology and indications endorsed by the Japanese Society of Echocardiography. Eur J Echocardiogr 2011;12:167-205.

5. Voigt JU, Pedrizzetti G, Lysyansky P, Marwick TH, Houle H, Baumann R, Pedri S, Ito Y, Abe Y, Metz S, Song JH, Hamilton J, Sengupta PP, Kolias TJ, d'Hooge J, Aurigemma GP, Thomas JD, Badano LP. Definitions for a common standard for 2D speckle tracking echocardiography: consensus document of the EACVI/ ASE/Industry Task Force to standardize deformation imaging. J Am Soc Echocardiogr 2015;28:183-93.

6. Chu M, Qian L, Zhu M, Yao J, Xu D, Chen M. Circumferential strain rate to detect lipopolysaccharideinduced cardiac dysfunction: a speckle tracking echocardiography study. Quant Imaging Med Surg 2019;9:151-9.

7. Sarvari SI, Haugaa KH, Zahid W, Bendz B, Aakhus S, Aaberge L, Edvardsen T. Layer-specific quantification of myocardial deformation by strain echocardiography may reveal significant $\mathrm{CAD}$ in patients with non-ST-segment elevation acute coronary syndrome. JACC Cardiovasc Imaging 2013;6:535-44.

8. Biering-Sørensen T, Shah SJ, Anand I, Sweitzer N, Claggett B, Liu L, Pitt B, Pfeffer MA, Solomon SD, Shah AM. Prognostic importance of left ventricular mechanical dyssynchrony in heart failure with preserved ejection fraction. Eur J Heart Fail 2017;19:1043-52.

9. Haugaa KH, Grenne BL, Eek CH, Ersbøll M, Valeur N, Svendsen JH, Florian A, Sjøli B, Brunvand H, Køber L, Voigt JU, Desmet W, Smiseth OA, Edvardsen T. Strain echocardiography improves risk prediction of ventricular arrhythmias after myocardial infarction. JACC Cardiovasc Imaging 2013;6:841-50.

10. Petri M, Orbai AM, Alarcón GS, Gordon C, Merrill JT, Fortin PR, Bruce IN, Isenberg D, Wallace DJ, Nived O, Sturfelt G, Ramsey-Goldman R, Bae SC, Hanly JG, Sánchez-Guerrero J, Clarke A, Aranow C, Manzi S, Urowitz M, Gladman D, Kalunian K, Costner M, Werth VP, Zoma A, Bernatsky S, Ruiz-Irastorza G, Khamashta MA, Jacobsen S, Buyon JP, Maddison P, Dooley MA, van Vollenhoven RF, Ginzler E, Stoll T, Peschken C, Jorizzo JL, Callen JP, Lim SS, Fessler BJ, Inanc M, Kamen DL, Rahman A, Steinsson K, Franks AG Jr, Sigler L, Hameed S, Fang H, Pham N, Brey R, Weisman MH, McGwin G Jr, Magder LS. Derivation and validation of the Systemic Lupus International Collaborating Clinics Classification Criteria for systemic lupus erythematosus. Arthritis Rheum 2012;64:2677-86.

11. Tektonidou MG, Andreoli L, Limper M, Amoura Z, Cervera R, Costedoat-Chalumeau N, Cuadrado MJ, Dörner T, Ferrer-Oliveras R, Hambly K, Khamashta MA, King J, Marchiori F, Meroni PL, Mosca M, Pengo V, Raio L, Ruiz-Irastorza G, Shoenfeld Y, Stojanovich L, Svenungsson E, Wahl D, Tincani A, Ward MM. EULAR Recommendations for the Management of Antiphospholipid Syndrome in Adults. Ann Rheum Dis 2019;78:1296-304.

12. Nagueh SF, Smiseth OA, Appleton CP, Byrd BF 3rd, Dokainish H, Edvardsen T, Flachskampf FA, Gillebert TC, Klein AL, Lancellotti P, Marino P, Oh JK, Popescu BA, Waggoner AD. Recommendations for the Evaluation of Left Ventricular Diastolic Function by Echocardiography: An Update from the American Society of Echocardiography and the European Association of Cardiovascular Imaging. J Am Soc Echocardiogr 2016;29:277-314.

13. Ozawa K, Funabashi N, Sugiura A, Kobayashi Y. Layer specific strain measurement and its relationship to heart failure indicators in systemic autoimmune disorder patients: A multi-layer transthoracic echocardiography study. Int J Cardiol 2016;220:693-9.

14. Carlsson M, Heiberg E, Ostenfeld E, Steding-Ehrenborg K, Kovács SJ, Flachskampf F, Arheden H. Functional Contribution of Circumferential Versus Longitudinal 
Strain: Different Concepts Suggest Conflicting Results. J Am Coll Cardiol 2018;71:254-5.

15. Stokke TM, Hasselberg NE, Smedsrud MK, Sarvari SI, Haugaa KH, Smiseth OA, Edvardsen T, Remme EW. Geometry as a Confounder When Assessing Ventricular Systolic Function: Comparison Between Ejection Fraction and Strain. J Am Coll Cardiol 2017;70:942-54.

16. Kraigher-Krainer E, Shah AM, Gupta DK, Santos A, Claggett B, Pieske B, Zile MR, Voors AA, Lefkowitz MP, Packer M, McMurray JJ, Solomon SD;PARAMOUNT Investigators. Impaired systolic function by strain imaging in heart failure with preserved ejection fraction. J Am Coll Cardiol 2014;63:447-56.

17. Cianciulli TF, Saccheri MC, Rísolo MA, Lax JA, Méndez RJ, Morita LA, Beck MA, Kazelián LR. Mechanical dispersion in Fabry disease assessed with speckle tracking echocardiography. Echocardiography 2020;37:293-301.

18. Lavall D, Kuprat LK, Kandels J, Stöbe S, Hagendorff A, Laufs U. Left ventricular mechanical dispersion in flowgradient patterns of severe aortic stenosis with narrow QRS complex. Int J Cardiovasc Imaging 2020;36:605-14.

19. Leong DP, Hoogslag GE, Piers SR, Höke U, Thijssen J, Marsan NA, Schalij MJ, Zeppenfeld K, Bax JJ, Delgado V. The relationship between time from myocardial infarction, left ventricular dyssynchrony, and the risk for ventricular arrhythmia: speckle-tracking echocardiographic analysis. J Am Soc Echocardiogr 2015;28:470-7.

20. Fudim M, Fathallah M, Shaw LK, Liu PR, James O, Samad Z, Piccini JP, Hess PL, Borges-Neto S. The Prognostic Value of Diastolic and Systolic Mechanical Left Ventricular Dyssynchrony Among Patients With Coronary Heart Disease. JACC Cardiovasc Imaging 2019;12:1215-26.

21. Chen J, Kalogeropoulos AP, Verdes L, Butler J, Garcia EV. Left-ventricular systolic and diastolic dyssynchrony as assessed by multi-harmonic phase analysis of gated SPECT myocardial perfusion imaging in patients with end-stage renal disease and normal LVEF. J Nucl Cardiol 2011;18:299-308.

22. Sanghera C, Wong LM, Panahi M, Sintou A, Hasham M,

Cite this article as: Luo T, Wang Z, Chen Z, Yu E, Fang C. Layer-specific strain and dyssynchrony index alteration in newonset systemic lupus erythematosus patients without cardiac symptoms. Quant Imaging Med Surg 2021;11(4):1271-1283. doi: 10.21037/qims-20-859
Sattler S. Cardiac phenotype in mouse models of systemic autoimmunity. Dis Model Mech 2019;12:dmm036947.

23. Bessant R, Duncan R, Ambler G, Swanton J, Isenberg DA, Gordon C, Rahman A. Prevalence of Conventional and Lupus-Specific Risk Factors for Cardiovascular Disease in Patients With Systemic Lupus Erythematosus: A CaseControl Study. Arthritis Rheum 2006;55:892-9.

24. Roman MJ, Crow MK, Lockshin MD, Devereux RB, Paget SA, Sammaritano L, Levine DM, Davis A, Salmon JE. Rate and determinants of progression of atherosclerosis in systemic lupus erythematosus. Arthritis Rheum 2007;56:3412-9.

25. Skaggs BJ, Hahn BH, McMahon M. Accelerated atherosclerosis in patients with SLE-mechanisms and management. Nat Rev Rheumatol 2012;8:214-23.

26. McHugh J. Systemic lupus erythematosus: Atherosclerosis confined to patients with nephritis. Nat Rev Rheumatol 2017;13:322.

27. Labonte AC, Kegerreis B, Geraci NS, Bachali P, Madamanchi S, Robl R, Catalina MD, Lipsky PE, Grammer AC. Identification of alterations in macrophage activation associated with disease activity in systemic lupus erythematosus. PLoS One 2018;13:e0208132.

28. Benagiano M, Borghi MO, Romagnoli J, Mahler M, Bella CD, Grassi A, Capitani N, Emmi G, Troilo A, Silvestri E, Emmi L, Alnwaisri H, Bitetti J, Tapinassi S, Prisco D, Baldari CT, Meroni PL, D'Elios MM. Interleukin-17/Interleukin-21 and Interferon- $\gamma$ producing $T$ cells specific for $\beta 2$ Glycoprotein I in atherosclerosis inflammation of systemic lupus erythematosus patients with antiphospholipid syndrome. Haematologica 2019;104:2519-27.

29. Gustafsson JT, Herlitz Lindberg M, Gunnarsson I, Pettersson S, Elvin K, Öhrvik J, Larsson A, JensenUrstad K, Svenungsson E. Excess atherosclerosis in systemic lupus erythematosus, -A matter of renal involvement: Case control study of 281 SLE patients and 281 individually matched population controls. PLoS One 2017;12:e174572. 